\title{
\#MeTooPoliSci: Addressing Gender Discrimination in Political Science
}

\section{Amanda Grigg, Associate Director, the Centennial Center for Political Science and Public Affairs}

$\mathrm{R}$ esearch continues to document the underrepresentation of women in faculty positions in political science and in top journals. Women who hold faculty positions in political science are more likely than male colleagues to experience discrimination and to rate their departments as intolerant. Women in the field are also more likely than men to face gender bias in their teaching.

Additionally, the problem of sexual harassment is pervasive in academia. A recent study commissioned by the Association of American Universities found that almost $70 \%$ of female graduate students reported sexual harassment on campus. In the world of political science, a 2017 APSA-commissioned study found that $30 \%$ of women respondents had experienced sexual harassment at APSA Annual Meetings between 2013 and 2016.

To address these issues, this year the Women's Caucus for Political Science held the first-ever \#MeTooPoliSci short course at the 2018 APSA Annual Meeting. The \#MeTooPoliSci course was designed, first and foremost, to create an empowering space in which women + could discuss their experiences of gender discrimination in the discipline. It also aimed to begin developing a set of specific policy recommendations for professional organizations, strategies for developing networks of allies to improve the disciplinary culture, and tools for localized action that participants could take back to their home institutions. Panels were facilitated by staff from the Boston Area Rape Crisis Center, and Greene and Hafer Employment Law, in addition to faculty members.

Following the event, co-organizer, the then-president of the caucus Nadia E. Brown described some of the insights gained during the short course. "We all had a collective aha moment when both the Boston Area Rape Crisis Center and the Law Firm said legal remedies are not the answer, litigation is the last resort. They described the ways in which Title IX and Title VII have helped us make progress, but can only take us so far. So it's up to us to do some of the heavy lifting that changes the culture that law can't do or that lawsuits can't do."

In their proposal and in the short course's programming, organizers also highlighted the importance of recognizing that the problems of gender bias can be compounded by other types of discrimination. Although fewer studies track the gendered experiences of $\mathrm{LGBTQ}+$ people in political science, evidence suggests that gender bias impacts their careers in negative ways. Similarly, race intersects with gender to create a unique hostile climate for women+ of color in political science. This environment was highlighted in a recent Washington Post article in which in Meg Guliford, a black woman and $\mathrm{PhD}$ candidate in international affairs, described repeatedly being confused with hotel staff at an academic conference. Research has also documented women+ with disabilities experiencing multiple and intersecting forms of harassment and exclusion. These same biases also work to undermine and devalue research focused on race, gender, sexuality, and disability.

Encouragingly, Brown also saw real promise in the group who gathered at the short course, and in APSA's dedication to addressing issues of gender inequality. She explained of organizing the event as a feminist collective, "There were growing pains but we learned and grew and the people that have stayed connected with the work have been warm and welcoming and have seen this as a collective of people that want to make the discipline better...it really was "leaderful" rather than being top-down." Brown also noted her excitement at APSA's active participation in efforts to promote gender equality in the discipline saying, "I don't know if that was true in the past, but it definitely is the future."

As for the future of \#MeTooPoliticalScience, their work is far from over. The Women's Caucus for Political Science is working to develop best practices to curtail sexual harassment in the discipline, working with regional caucuses to hold short courses similar to the pilot held at the annual meeting, and working on ADVANCE partnership grants for National Science Foundation support for their continued efforts.

If you're interested in learning more about the Women's Caucus for Political Science, the \#MeTooPoliticalScience project, or the Special Projects Fund please contact centennial@apsanet.org. To learn about APSA's efforts to support diversity and inclusion in the profession, visit https:// www.apsanet.org/diversityprograms.

The project's PIs are: Nadia E. Brown (Purdue University), Rebecca Gill (The University of Nevada, Las Vegas), Jennifer Merolla (The University of California, Riverside), Melissa R. Michelson (Menlo College), Elizabeth Sharrow (The University of Massachusetts Amherst), Patricia Stapleton (Worcester Polytechnic Institute), and Dara Strolovitch (Princeton University).

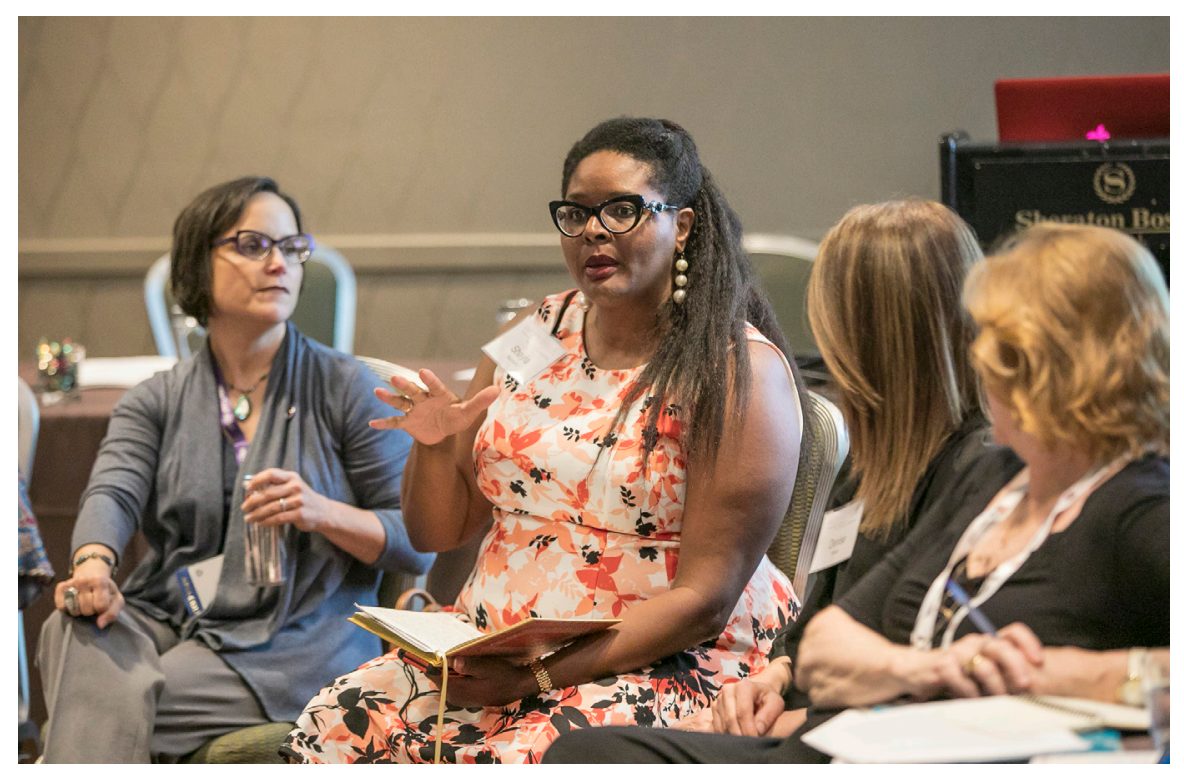

Shayla Nunnally, center, speaks, at the Women's Caucus for Political Science's first-ever \#MeTooPoliSci short course at the 2018 APSA Annual Meeting. 